\title{
Ear-Lead Multiple Smart Bio Sensor System in M-health
}

\author{
Numan Celik \\ Brunel University London \\ Department of Electronic and \\ Computer Engineering \\ Uxbridge, UB8 3PH, London, UK \\ +441895 274000 \\ numan.celik@brunel.ac.uk
}

\author{
Wamadeva Balachandran \\ Brunel University London \\ Department of Electronic and \\ Computer Engineering \\ Uxbridge, UB8 3PH, London, UK \\ +441895 265774 \\ Nadarajah Manivannan \\ Brunel University London \\ Department of Electronic and \\ Computer Engineering \\ Uxbridge, UB8 3PH, London, UK \\ +441895 274000 \\ Wamadeva.Balachandran@bru Nadarajah.Manivannan@brunel \\ nel.ac.uk
}

\begin{abstract}
In this paper, we present a multi-parameter wearable sensor system in conjunction with a smartphone to enable a real-time unobtrusive monitoring of core body temperature, electrocardiogram (ear-lead ECG), and blood oxygen saturation $\left(\mathrm{S}_{\mathrm{p}} \mathrm{O}_{2}\right)$ on ambulatory patients. Clinical research illustrating that continuing accurate measurements of core body temperature (CBT) are crucial to investigate human thermoregulation in ambulatory environment and during activity. On the other hand, ECG remains the mainstay test for primary diagnosis and survival analysis of heart diseases. We implement a wireless multisensory system that measures both the tympanic temperature inside the ear and ECG data from an ear and an arm. The behind-the-Ear device makes desirable to measure ECG data without any chest belt and allow the patient to move around freely. The proposed Bluetooth device has similarity of a hearing aid and is wirelessly connected to a smartphone for data transmission and displaying. This device not only gives access to the core temperature and ECG data in real time, but also the device can be controlled - removed and reapplied - by the patient at any time, thus increasing the performance of personal health applications.
\end{abstract}

\section{Categories and Subject Descriptors}

B.4 [Input Output and Data Communications]: Data Communication Devices; B.7 [Integrated Circuits]: Miscellaneous; J.3 [Life and Medical Sciences]: Medical Information Systems.

\section{General Terms}

Measurement, Performance, Design, Experimentation.

\section{Keywords}

Wireless Body Area Networks (WBAN), ECG, Core Body Temperature, Oxygen Saturation Level $\left(\mathrm{SpO}_{2}\right)$, Sensor Fusion, Wearable Computing.

\section{INTRODUCTION}

The rapid growth of wireless technologies brings new innovative ideas that enables continuous real-time remote patient monitoring of evolving healthcare services within compact wireless body sensors. The services and technologies provide relatively uncontroversial, well-communicated and monitoring devices, developed to give more affordable solutions specifically for mobile healthcare, such as daily activity monitoring, personal healthcare and monitoring systems, and body sensor systems that can alarm the clinicians via the patients' mobile phones. New trend in remote patient monitoring is moving toward the use of personal mobile devices compatible with multiple biomedical sensors using Bluetooth wireless communication [1].

In practice, this recent mobile health (m-Health) technology enables to see the people's daily activity in their smartphones. In addition, these mobile-based portable embedded devices will provide platforms to monitor their critical biological data continuously and remotely. An assessment report has been prepared for the European Union regarding the effectiveness of $\mathrm{m}$ Health in biomedical applications and the diagnosis of the diseases in 2013. According to this report, m-Health could save 99 billion Euros in healthcare costs in the EU and add 93 billion Euros to the EU GDP in 2017 if its adoption is encouraged [2].

Critical body measurements such as heart rate, blood pressure, blood glucose, core temperature, ECG, EEG and oxygen saturation level $\left(\mathrm{SpO}_{2}\right)$ are truly key factors in determining the overall health of a patient. Continuous remote monitoring of these critical signs can play an important role to identify patient's disease, in particular for epilepsy, heart attack, diabetes, sleep apnea, and heart failure [3].

Electro-cardiogram (ECG) is a critical signal from the heart which may be measured from two or more sensors placed on the patient's skin. ECG monitoring is a basic diagnosis procedure to figure out whether the patients have heart diseases. Core body temperature (CBT) is a known physiological indicator of the human body. It is closely linked to a number of clinically diagnosed diseases, particularly in the characterization and diagnosis of human sleep disorders to estimate the circadian-system, which is 24-hour cycle in biological processes [4], and also CBT monitoring is a wide area of research for human thermoregulation applications. Pulse 
oximetry is another vital sign for diagnosing patients' diseases such as sleep apnea and is measured using PPG (photoplethysmography) techniques (see Figure 1).

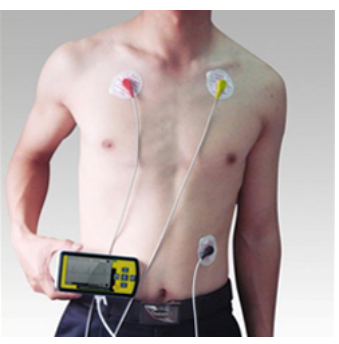

a)

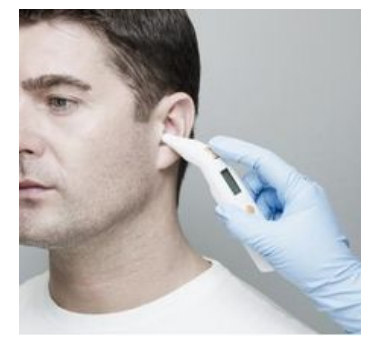

b)

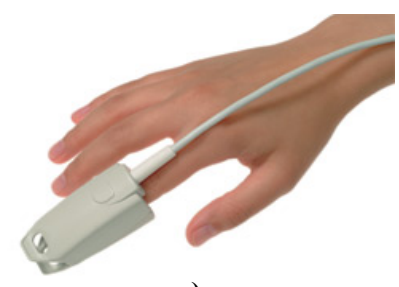

c)

Figure 1. a) the typical 3-lead ECG monitoring system. b) eartympanic device to measure core body temperature. c) the reflective PPG sensor to measure blood oxygen saturation (SpO2) and heart rate.

Here, we examine a continuous, wearable and wireless criticalsign monitor worn behind the ear, combining these 3 (ECG, CBT, and PPG) sensors. The reason for choosing the ear as a location in these experiments is that it makes possible to measure ECG, CBT, and blood oxygen discreetly since the sensors can be partially hidden by hair and the ear.

This paper aims to advance the ambulatory ECG treatments by proposing a wearable behind-the-ear smart sensor that transmits integrated biological data wirelessly to a smartphone and monitors ECG, $\mathrm{CBT}$ and $\mathrm{SpO}_{2}$ information in real-time. Since most conventional systems take each data separately and from different locations on the body. In contrast, we developed a smart sensor system that facilitates sensor fusion by sealing into an ear mold. Moreover, this design is less disrupted by motion artifacts. In the future, this option not only gives flexibility to the patient, but also gives the clinicians to access the physiological data in rea time.

\section{RELATED WORK}

When a ubiquitous smart sensor is developed, there should be basic components that should be considered: easy-for-use, mobility, accuracy, and security. There are currently many ongoing researches that investigate various solutions on the design of wireless personal healthcare monitoring devices. Lee et al. developed a mobile phone based ECG monitoring application. The system describes the design and implementation of a prototype tele-health system which monitored physiological signals of patients in real-time [5]. Sanches et al. designed an electronic temperature sensor within a headset Bluetooth device that sends the temperature measurements to a mobile phone. The proposed system measures central body temperature continuously at the ear [6]. Jung et al. proposed a wireless body sensor platform called 'Virtual Cuff' that comprises PPG and ECG sensors to estimate systolic and diastolic blood pressure (BP). The presented work fuses data from various sensors, including ECG, PPG, accelerometer, and GPS, for extrapolating BP information using signal characteristics that are derived from PPG and ECG waveforms [7]. Do Valle et al. examined [8] a behind-the-ear device that records EEG measurements on smartphone continuously and then uploads the patients' data to a secure server. Song et al. developed a body monitoring system design based on android smartphone including three main functions such as brainwave capture (EEG), ECG and temperature. These data are gathered by hardware and sent to the Bluetooth receiving device of android smartphone [9]. Boano et al. managed to measure core body temperature (CBT) on ambulatory patients and exercising athletes using a wireless wearable device that measures the tympanic temperature at the ear. The CBT data is transmitted via ATmega128RFA1 chip based on ZigBee communication which is different from other studies [10].

The brief summary of the literature review illustrate the use of modern communication technology for data exchange between ambulatory patients and mobile devices by combining several sensors in one typical system. This is one of the key factors to make m-Health platform much more desirable. However, not only for clinical purposes, but also an integrated and personalized smart sensor device, easy to use and less affected motion artifacts.

\section{SYSTEM ARCHITECTURE OVERVIEW}

The smart sensor platform was developed to experimentally combine 3 different body sensors and monitor vital parameters on the smartphone. The main characteristics of the proposed system are to integrate and monitor ear-lead ECG, ear-lead core temperature, oxygen saturation level (PPG from the finger) and to communicate to mobile client by using Bluetooth connection. The ECG and tympanic sensors for displaying the physiological data are integrated in the form of an earbud. PPG sensor will be attached onto the finger and it will be connected to the microcontroller unit (MCU). Each biopotentials will be experimentally examined in Subsection 3.1 and fusion of sensors will be analysed in Subsection 3.2, respectively.

\subsection{Sensor Data Acquisition System}

Here, each of the biosensors will be separately examined within the system design prototypes. Our system comprises both hardware and software units.

- The hardware part of the system: The single microcomputer system (Arduino UNO), a tympanic infrared sensor detecting core temperature module MLX90614, an ECG acquisition circuit module integrated with Arduino, a pulse oximeter detecting module CMS50EW, a wireless Bluetooth device, and an Android based smartphone. Figure 2 shows a block diagram to describe the multi-parameter body monitoring system using a smartphone.

- The software part of the system: Arduino IDE is used to control different hardware pieces of the sensors and communicate with Bluetooth equipment. Application programs of android platform based on the Java language were tested and implemented by using Android Studio. The installed software can begin the Bluetooth connection between the smartphone and the hardware unit, thus the biological data can be transferred to the smartphone. 


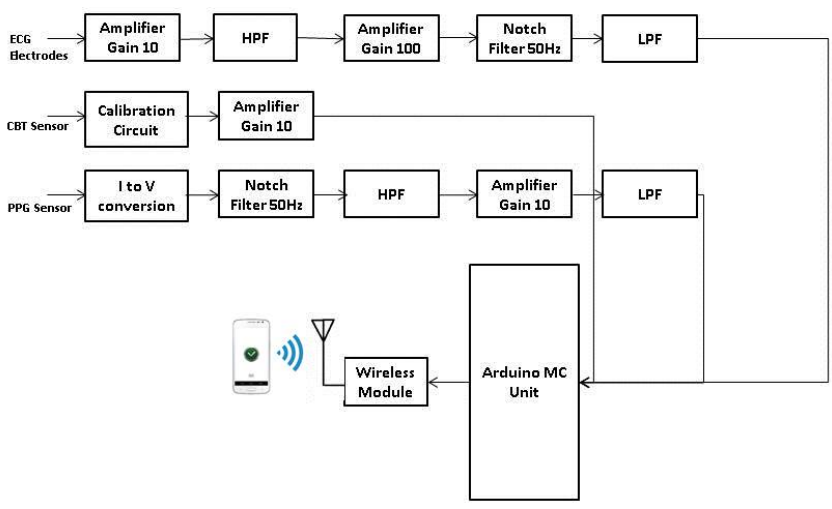

Figure 2. The block diagram of body monitoring system design

\subsubsection{ECG Acquisition Platform}

The core of ECG sensor platform as shown in Figure 3, is based on the e-Health sensor shield. The e-Health sensor shield allows performing in conjunction with Arduino. This enables to combine different biosignals in Arduino. The sensor shield gets ECG data and transmits through Arduino UNO. The system consists of an analog amplifier module with one differential (instrumentation) amplifier and two general purpose operational amplifiers. The signal quality is mostly determined by the instrumentation amplifier (IA) due to having low noise amplifying unit with low power dissipation. The operational amplifiers are used to forward the low voltage to a base line for signal processing with a gain of 100. The ECG signals are restricted within the range of bandwidth of $0.5-100 \mathrm{~Hz}$. High and low pass filters were used after the first and second stages of amplification, respectively. The ECG signal is sampled at $2,048 \mathrm{~Hz}$ and digitized using a 16 bit ADC. The filtered digital signal is then decimated to reduce the rate of samples from $2,048 \mathrm{~Hz}$ down to $256 \mathrm{~Hz}$ and then transferred to an Android based smartphone via our Bluetooth module. The principle of data transmission has been developed in the same way after the signal processing of each sensor (CBT and PPG sensors).
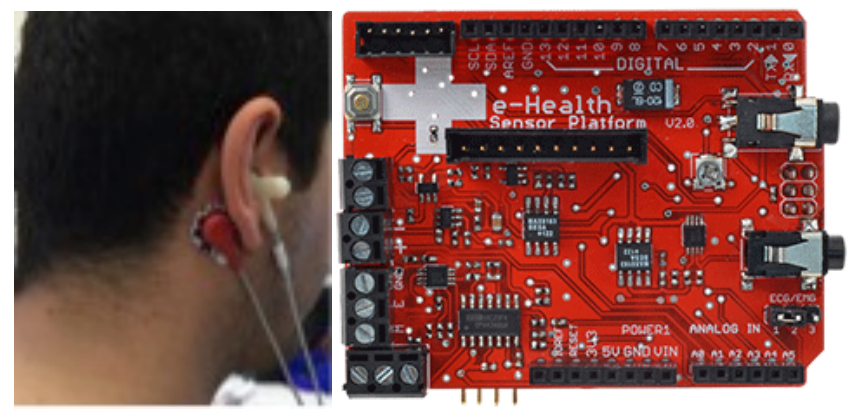

Figure 3. Earable ecg sensor placement (left); Electronic circuit for ECG amplification (right)

\subsubsection{CBT Sensing Platform}

In our proposed system we have used a thermopile sensor that is a non-contact sensor for measuring core temperature. Because tympanic temperature directly reflects the core temperature of the carotid artery [11], we have proposed an earbud-design infrared tympanic sensor that can continuously measure the temperature of the tympanic membrane (Figure 4). This design connects with our Arduino MCU, which is the core controller of the whole smart sensor system, to perform amplification, filtering and ADC processes. The thermopile sensor (MLX90614) gives us very sensitive information regarding the core body temperature with 17 bit resolution, thus this could be in some cases $0.0034{ }^{\circ} \mathrm{C}$ resolution.

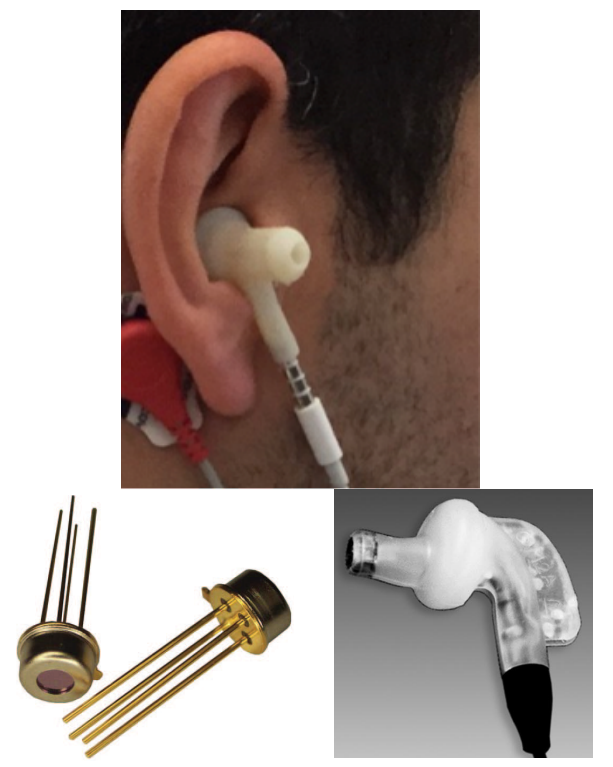

Figure 4. Our proposed earphone-type infrared sensor (above); Infrared thermopile sensor (below-left), another view of earphone-embedded thermopile sensor (below-right)

\subsubsection{Pulse Oximetry Acquisition}

In general, the pulse oximeter consists of a transductor which initiates two LEDs and a photodiode detector. One of the LEDs emits red light (with a wavelength of $\lambda=660 \mathrm{~nm}$ ) and other LED performs an infrared light $(\lambda=940 \mathrm{~nm})$. This technique is known as PPG (photoplethysmography) and the PPG sensor is based on the theory that the color of blood range depends on the oxygen it contains. For instance, hemoglobin particularly reflect more red lights, and PPG sensor decides oxygen saturation in the blood $\left(\mathrm{SpO}_{2}\right)$ according to this volume. For our test-bed we have performed this test using Amperor Bluetooth Finger Pulse Oximeter as that shown in Figure 5. The smart sensor system incorporates a Bluetooth connection that enables to gather PPG data from this pulse oximeter sensor. The sensor is powered with a rechargeable lithium battery lasting for about 20 hours in continuous mode. A $50 \mathrm{~Hz}$ notch filter is used for both ECG and PPG signals to eliminate the power line interference, thus there would not be any loss at $50 \mathrm{~Hz}$ component of the signals.

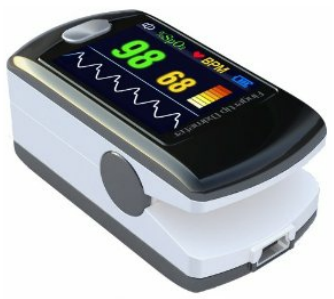

Figure 5. Bluetooth pulse oximeter

\subsection{Sensor Fusion}

After digitizing and conditioning the analog signals from each sensor, the suitable digital biological data are MUXed at the data acquisition hardware unit in an appropriate way. The data after being acquired by Arduino is converted in the form of packets and is wirelessly transmitted to Android based mobile phone. Figure 6 indicates ECG electrodes placement and appearance of general prototype of performed body sensors. 


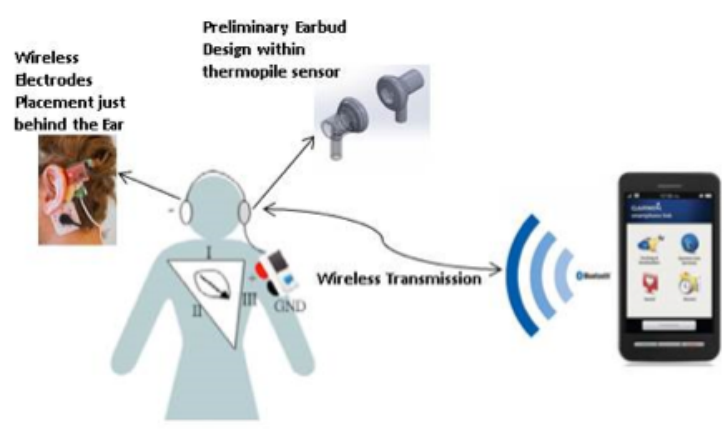

Figure 6. Ear-lead wearable multiple sensor monitoring system

\subsection{Bluetooth}

Bluetooth is a very useful technology to communicate wirelessly in short-range applications such as exchanging data from short distance fixed mobile devices. With the characteristic of synchronization with other Bluetooth devices, the wireless body area network can be applied successfully. Our proposed system uses HC-05 Bluetooth module that consists of different modes in its processing system. Figure 7 shows our Bluetooth serial module, which is paired to the smartphone.

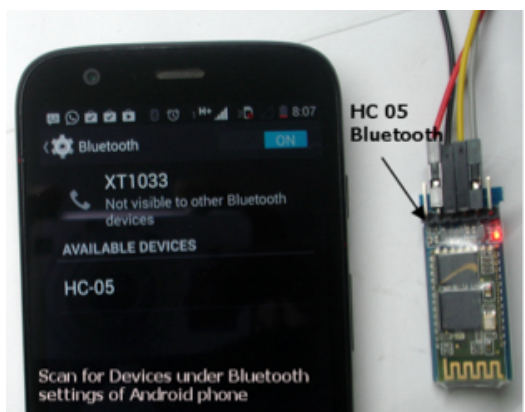

Figure 7. The view of Bluetooth module while pairing with the smartphone

As mentioned, there are different channels (ECG, CBT, and PPG) that need to be transmitted through Bluetooth communication. The communication module has the capability to transmit data over the Bluetooth with maximum baud rate of $115,200 \mathrm{bps}$. We have to calculate the overall bandwidth before transmitting the biological data. For our system, we have developed three different sensing parameters (ECG, CBT, and PPG). Core body temperature (CBT) measurement operates at $20 \mathrm{~Hz}$ sampling frequency (16-bit samples). ECG sensor works at $250 \mathrm{~Hz}$ frequency range (16-bit samples) with 3 electrodes and PPG operating at a $30 \mathrm{~Hz}$ (16-bit samples) with two photodiodes. Hence, the total data rate will comprise from aggregating each measured signal's bit rates. The total minimum bandwidth of the system will be:

Baud Rate $=$ bit rate of $($ CBT $(\mathrm{kbps})+$ ECG $(\mathrm{kbps})+$ PPG (kbps))

$\mathrm{BR}=$ (sampling frequency of $\mathrm{CBT}) *$ (Nyquist-criteria) $*$ (sampling bit number) + (sampling frequency of ECG) * (Nyquistcriteria) * (sampling bit number) * (used electrodes) + (sampling frequency of PPG) * (Nyquist criteria) * (sampling bit number) * (used electrodes)

$\mathrm{BR}=20 * 2$ (Nyquist-criteria) $* 16+250 * 2 * 16 * 3+30 * 2 *$ $16 * 2=0.64+24+1.92=26.56 \mathrm{kbps}$.

The above number is much less than the overall Bluetooth transmission baud rate at 115,200 bps. Thus, the residue bandwidth would be enough to perform two-way handshaking and sending the data successfully.

\section{EXPERIMENTS and RESULTS}

Several experiments were conducted with one wireless multiparameter body monitoring system. The analysis and display of ECG, CBT and $\mathrm{SpO}_{2}$ was done firstly in a $\mathrm{PC}$, then in a mobile phone via a Bluetooth module transmitter.

Regarding the ECG analysis, traditional $\mathrm{Ag} / \mathrm{AgCl}$ electrodes (Ambu) were used to get ECG signal from the ear and the electrodes were placed just behind the ear. ECG results were displayed in a PC primarily using KST real-time data viewing plotting tool. After that, the ECG data was monitored in Android based smartphone using Android Studio software program and Arduino IDE software tools. Both results are shown together in Figure 8.

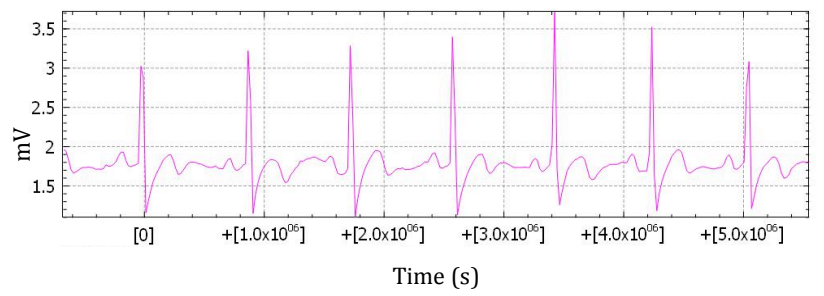

a)

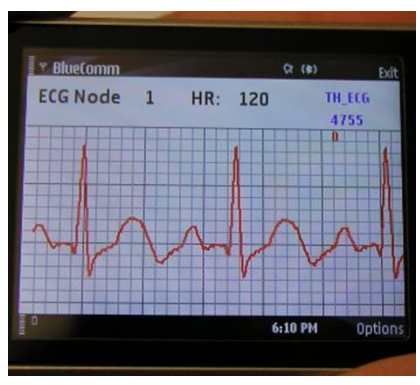

b)

Figure 8. ECG Results. a) ECG data in PC - KST platform. b) ECG data in Android based mobile phone - Samsung Galaxy Ace

The second experiment aimed at detecting core body temperature from tympanic membrane at the ear. Figure 9 illustrates the CBT monitoring in the ear for 100 seconds in Matlab environment, and mobile phone, respectively.

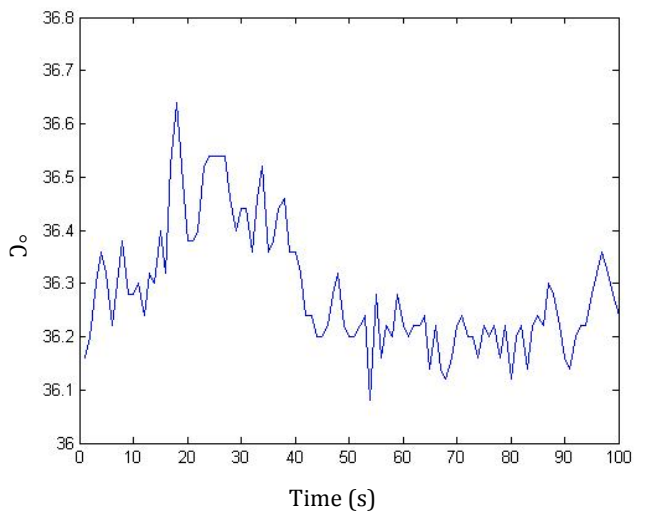

a) 


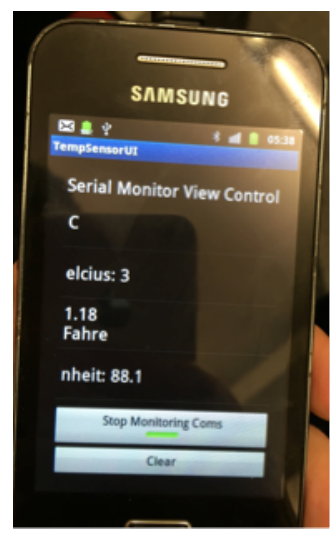

b)

Figure 9. CBT Monitoring on Matlab while plotting the data a); and on mobile device as giving Celsius and Fahrenheit degrees (b).

As can be seen in the graph, the core temperature varies between $36.1^{\circ} \mathrm{C}$ and $36.65^{\circ} \mathrm{C}$ in the ear.

The final experiment results obtained for oxygen saturation level in the blood $\left(\mathrm{SpO}_{2}\right)$ can be seen in Figure 10. The level of $\mathrm{SpO}_{2}$ is at around $98 \%$ in the blood. The PPG data is shown in the picture as well as the $\mathrm{SpO}_{2}$ ratio.

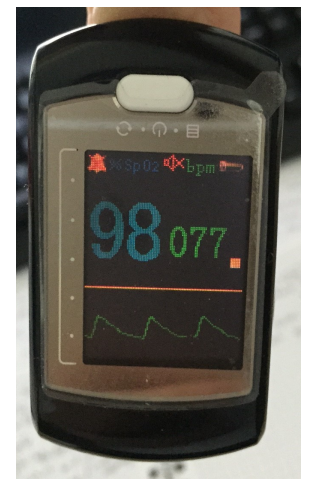

Figure 10. Oxygen saturation level in blood within PPG data using Amperor Bluetooth pulse oximeter device

\section{CONCLUSION}

We have proposed an ear-lead multi-parameter smart sensor system that acquires different physiological information and continuously monitor an Android based smartphone, giving patients real time control of the data. This device includes nonintrusive sensors, specifically ECG, CBT and PPG with high accuracy. Furthermore, it facilitates the difficulties of wearables giving patients significantly less restriction by eliminating the need for adapting intrusive equipment or using a laptop to see the biological data. In the future, this smart sensor system can work with a wide body area network system to offer accessing patients data by clinicians and healthcare professionals to get more accurate and quicker information for the diagnosis and treatment options.

\section{REFERENCES}

[1] Bifulco, P., Cesarelli, M., Fratini, A., Ruffo, M., Pasquariello, G., and Gargiulo, G. 2011. A wearable device for recording of biopotentials and body movements. In Proceedings of the IEEE International Symposium on Medical Measurements and Applications (Bari, Italy, May 30 - 31, 2011). DOI= http://doi.org/10.1109/MeMeA.2011.5966735

[2] "Socio-economic impact of mHealth - An assessment report for the European Union". GSMA Connected Living Programme. June 2013. Web. 21.05.2014

http:/www.gsma.com/connectedliving/wpcontent/uploads/2013/06/Socio-economic impact-ofmHealth EU 14062013V2.pdf

[3] Pierdomenico, D. S., Nicola, D. M., Esposito, L. A., et al. 2009. Prognostic value of different indices of blood pressure variability in hypertensive patients. American journal of hypertension. 22, 8 (June. 2009), 842-847. DOI= http://doi.org/10.1038/ajh.2009.103

[4] Scheer, F. A. J. L., Kenneth, P. W., Richard, E. K., Charles A. C., 2007. Plasticity of the intrinsic period of the human circadian timing system. PloS ONE, 2007. DOI= doi:10.1371/journal.pone.0000721

[5] Lee, D. H., Choi, J., Rabbi, A., Fazel-Rezai, R., 2012. Development of a mobile phone based e-Health monitoring application. International journal of advanced computer science and applications. 3, 3 (2012), 38-43. DOI= http://dx.doi.org/10.14569/IJACSA.2012.030307\#sthash.fZX kf8zk.dpuf

[6] Sanches, J. M., Pereira, B., Paiva, T., Headset Bluetooth and cell phone based continuous central body temperature measurement system. In Proceedings of the IEEE Engineering in Medicine and Biology Society (Buenos Aires, Argentina, August 31 - Sept 4, 2010). DOI= http://doi.org/10.1109/IEMBS.2010.5626172

[7] Jung, K. H., Tran, V., Gabrielian, V., and Nahapetian, A., 2014. Virtual cuff: multisensory non-intrusive blood pressure monitoring. In Proceedings of the 9th International Conference on Body Area Networks (BodyNets '14). DOI= http://dx.doi.org/10.4108/icst.bodynets.2014.257046

[8] Do Valle, B. G., Cash S. S., and Sodini C. G., Wireless behind-the-ear eeg recording device with wireless interface to a mobile device (iphone/ipod touch). In Proceedings of the IEEE Engineering in Medicine and Biology Society (Chicago, US, August 26-30, 2014). DOI= 10.1109/EMBC.2014.6944984

[9] Song, W., Yu, H., Liang, C., Wang, Q., and Shi, Y., Body monitoring system design based on android smartphone. In Proceedings of the 2012 World Congress on Information and Communication Technologies, WICT 2012. DOI= http://doi.org/10.1109/WICT.2012.6409247

[10] Boano, C. A., Lasagni, M., and Romer, K., Non-Invasive measurement of core body temperature in marathon runners. In Proceedings of the Body Sensor Networks 2013 (BSN '13) (Cambridge, US, May 6-9, 2013). DOI= 10.1109/BSN.2013.6575484

[11] Bock, M., Hohlfeld, U., von Engleln, K., Meier, P. A., Motsch, J., Tasman, A. J., The accuracy of a new infrared ear thermometer in patients undergoing cardiac surgery, Anesthesia \& Analgesia. $105-6,2007$. DOI= 10.1213/01.ane.0000289639.87836.79 